\title{
Distribution of therapeutic response in asthma control between oral montelukast and inhaled beclomethasone
}

\author{
R.A. Baumgartner*, G. Martinez ${ }^{\#}$, J.M. Edelman ${ }^{\star}$, G.G. Rodriguez Gomez ${ }^{+}, M^{*}$ Bernstein ${ }^{\S}$, \\ S. Bird", R. Angner*, A. Polis ${ }^{\uparrow}$, S.B. Dass*, S. Lu*, T.F. Reiss*, \\ for the Montelukast Asthma Study Group
}

Distribution of therapeutic response in asthma control between oral montelukast and inhaled beclomethasone. R.A. Baumgartner, G. Martinez, J.M. Edelman, G.G. Rodriguez Gomez, M. Bernstein, S. Bird, R. Angner, A. Polis, S.B. Dass, S. Lu, T.F. Reiss, for the Montelukast Asthma Study Group. (C) ERS Journals Ltd 2003.

ABSTRACT: The distribution of responses in study populations provides a novel method of comparing the benefit of two treatments. This 6-week, randomised, placebocontrolled, double-blind study compared the effectiveness of oral montelukast with inhaled beclomethasone in chronic asthma by assessing the distribution and overlap of patient responses to therapy, as measured by a clinical outcome (asthma control days).

A total of 730 adult patients with asthma, age 15-65 yrs, with a forced expiratory volume in one second (FEV1) at baseline of $50-85 \%$ of predicted and $\geqslant 15 \%$ improvement in FEV1 after inhaled $\beta$-agonist were enrolled. After a 2-week placebo run-in period, patients were randomly allocated to receive montelukast $(10 \mathrm{mg}$ once daily), inhaled beclomethasone (200 $\mu \mathrm{g}$ twice daily) or placebo. The primary end-point (per cent of asthma control days) was compared between treatments as the overlap in the response distributions.

The overlap of the distribution of responses between the montelukast and beclomethasone groups was $89 \%$ for per cent asthma control days and $96 \%$ for change from baseline in FEV1. The mean $( \pm S D)$ per cent asthma control days in the montelukast and beclomethasone groups was significantly higher than that in the placebo group (placebo 40.0 \pm 35.8 , montelukast 50.7 \pm 37.1 , beclomethasone 57.9 \pm 36.1 ). The mean differences between montelukast and placebo, beclomethasone and placebo, and montelukast and beclomethasone were significant. The mean per cent change $( \pm$ SD) from baseline in FEV1 was $12.1 \pm 18.7$ and $13.9 \pm 20.8$ in the montelukast and beclomethasone groups, respectively, and significantly greater than that in the placebo group (6.4 \pm 20.1$)$; there was no significant difference between the montelukast and beclomethasone groups in mean values or response distribution. There was also no difference among treatment groups in the frequency of adverse experiences.

A comparison of the response distribution is an important approach to comparing therapies; montelukast and beclomethasone provided similar response distributions for the end-point of per cent asthma control days over a 6-week treatment period.

Eur Respir J 2003; 21: 123-128.
*Merck Research Laboratories, Merck \& Co., Inc., Rahway, NJ, USA ${ }^{\#}$ Clinicas Medicas, Guatemala City, Guatemala. "United States Human Health, Merck \& Co., Inc., Horsham, PA, USA. ${ }^{+}$Hospital Mexico, Instituto Costarricense de Investigaciones Clinicas, San Jose, Costa Rica. ${ }^{8}$ Hospital de Clinical Caracas, Caracas, Venezeula.

Correspondence: T.F. Reiss

Merck Research Laboratories

RY 34B-328

Rahway

NJ 07065-1900

USA

Fax: 17325947830

E-mail: theodore_reiss@merck.com

Keywords: Asthma asthma control days

beclomethasone

forced expiratory volume in one second leukotriene receptor antagonist montelukast

Received: April 82002

Accepted after revision: August 52002
Although the incidence of asthma has increased substantially over the last two decades in children and adults [1], major advances have also been made in understanding the pathophysiology of this chronic inflammatory disease. Cysteinyl leukotrienes, the products of inflammatory cells such as eosinophils and mast cells, are inflammatory mediators that play an important role in the pathophysiology of asthma [2-5]. Antileukotriene agents, including montelukast, zafirlukast, pranlukast and the 5-lipoxygenase inhibitor zileuton, act by blocking the effects of the cysteinyl leukotrienes. Montelukast is an orally active, potent and specific cysteinyl leukotriene receptor antagonist that has been shown to improve the signs and symptoms of asthma in several placebo-controlled trials of patients with chronic asthma $\geqslant 2$ yrs of age [6-9].
National and international consensus guidelines for treating asthma recommend daily controller therapy for patients with persistent asthma $[10,11]$. Inhaled corticosteroids are widely used as an initial controller therapy and antileukotriene agents are currently recommended as an alternative controller therapy. However, there are limited data directly comparing inhaled corticosteroids with antileukotrienes [8].

According to treatment guidelines, the aim of longterm therapy for asthma is to minimise symptoms, avoid asthma exacerbations and maintain normal physical activity $[10,11]$. However, therapeutic efficacy in most clinical trials is assessed by changes in surrogate end-points, such as spirometry and home peak flow monitoring. Instead, measures of more clinically relevant, patient-oriented outcomes, such as 
symptoms, need for rescue medication, and incidence of asthma exacerbations, may better reflect asthma control as defined by treatment guidelines [10, 11]. Additionally, most analysis of clinical trials rely on comparisons of mean values. Such data, however, frequently fail to reflect important clinical information present in the variability around the mean response, otherwise known as the distribution of response in the population. The overlap in response distributions can frequently provide a more complete determination of clinical comparability because the range of response to therapy of individuals in the study population is taken into account [12].

The purpose of this study was to compare the effectiveness of montelukast and inhaled beclomethasone in the treatment of adult patients with asthma by evaluating the response distributions for both conventional measurements such as airway function, as well as an outcome measure (the asthma control day) that incorporates symptoms, rescue medication use, and incidence of asthma attacks. The primary hypothesis of the study was that the overlap of patient response in the percentage of asthma control days between montelukast and beclomethasone would be $\geqslant 80 \%$. This value was chosen because it represents the commonly used level of assessing bioequivalence [13].

\section{Patients and methods}

\section{Patients}

Male and female patients aged $\geqslant 15$ yrs with $\geqslant 1-y r$ history of asthma symptoms were enrolled in the study. Patients were eligible for enrollment if they had a forced expiratory volume in one second (FEV1) at baseline of $50-85 \%$ of the predicted value, with $\geqslant 15 \%$ improvement in FEV1 after inhaled $\beta$-agonist and average daily use of more than two puffs of $\beta$-agonist during the run-in period. Eligible patients had a smoking history of $\leqslant 7$ pack-yrs and were nonsmokers for $\geqslant 1 \mathrm{yr}$. A total of 730 patients were randomised into the study.

During the study, patients continued to take a short-acting inhaled $\beta$-agonist as necessary to control asthma symptoms. Use of oral, intravenous and intramuscular corticosteroids was not permitted within 1 month of the start of the placebo run-in period. In addition, patients were excluded from the study if they had received inhaled corticosteroids within 2 weeks, astemizole within 3 months or xanthine derivatives, oral or long-acting inhaled $\beta$-agonists, cromolyn sodium or nedocromil, inhaled anticholinergic agents, oral leukotriene receptor antagonists or leukotriene synthesis inhibitors within 1 week before the start of the placebo run-in period. Patients could receive immunotherapy if it had started $>6$ months before the start of the placebo run-in period and if the dosage was expected to remain the same during the study. Females of childbearing potential were required to have a negative pregnancy test (serum $\beta$-human chorionic gonadotropin) prior to the start of the placebo run-in period. The study protocol was approved by local ethics review committees and all enrolled patients gave written informed consent to participate in the study.

\section{Study design}

This multicentre, double-blind, randomised, placeboand active-controlled, parallel-group study was conducted at 16 centres in eight countries (Canada, Brazil, Chile, Peru, Venezuela, Mexico, Costa Rica and Guatemala). There was a 2-week, single-blind, placebo (baseline) run-in period followed by a 6-week, double-blind active treatment period. Eligible patients were randomly assigned (in a 3:3:1 ratio of montelukast:beclomethasone:placebo), according to a computergenerated allocation schedule, to receive oral montelukast (10 mg once daily), inhaled beclomethasone $(200 \mu \mathrm{g}$ (four puffs) twice daily) or placebo for 6 weeks. Study medications were administered using a double-dummy regimen: montelukast or matching placebo was taken once daily at bedtime (irrespective of food) and beclomethasone or matching placebo inhaler was administered twice daily (spacer devices were permitted, but not required). Patients were evaluated every 2 weeks.

Spirometry was conducted between 06:00 and 10:00 $\mathrm{h}$ at each clinic visit using a standard spirometer (Model PB 100; Puritan-Bennett, Lenexa, KS, USA) in accordance with the reproducibility and acceptability criteria of the American Thoracic Society [14] and a standard quality control system [15]. The largest FEV1 and forced vital capacity from the three best acceptable manoeuvres were recorded.

A daily asthma diary card was completed by each patient. This diary card contained a daytime section that captured the total puffs of salbutamol taken since arising and a night-time section that captured nocturnal awakenings because of asthma and total puffs of salbutamol taken since bedtime. Daytime asthma symptoms were not recorded. The nocturnal awakening question has been shown to have acceptable measurement [16].

At the final study visit or at discontinuation, patients and physicians independently evaluated the change in the patients' asthma condition by selecting the most appropriate response from a seven-point global evaluation scale, ranging from very much better (score 0 ) to unchanged (score 3 ) to very much worse (score 6). When completing the physician global evaluation, the investigator was blinded to all study data except for the following items from the final visit: patients' oral history, vital signs, physical examination and spirometry results.

\section{Statistical analysis}

The primary end-point was the percentage of asthma control days during the study, analysed as the percentage overlap in the response distribution. Consistent with previous use of this end-point [17], an asthma control day was defined as a day when the patient used less than or equal to two puffs of inhaled salbutamol, had no nocturnal awakenings 
and experienced no asthma attack. An asthma attack occurred when a patient required unscheduled asthmarelated medical care or initiated use of oral corticosteroid rescue medication. Additional end-points included FEV1 (calculated as the change in absolute FEV1 from baseline), average daily inhaled shortacting $\beta$-agonist use, patient and physician global evaluations and incidence of asthma attacks.

An intention-to-treat approach was followed in the analysis of efficacy data. All patients with a baseline and at least one postrandomisation measurement were included in the analysis according to the group to which they were randomised. All randomised patients were included in the analysis of safety. The analysis of per cent overlap for the end-points of asthma control days and FEV1 was based on a standard nonparametric statistical method (Mann-Whitney U statistical version of the Wilcoxon rank test) that permits comparison of each individual in the beclomethasone group to each individual in the montelukast group; it was thus a patient-based rather than a populationbased measure [18-21]. The standardised MannWhitney $U$ statistic and its corresponding variance were used to calculate a 95\% confidence interval (CI) for the true probability that a randomly chosen patient in the beclomethasone group experienced a greater percentage of asthma control days than a randomly chosen patient in the montelukast group. Equivalence between the treatment groups would result in a value of 0.5 (i.e. a $50 \%$ probability). To facilitate the interpretation of results in terms of overlap in the distribution of percentage of asthma control days and thus the treatment difference, estimates and 95\% CI limits were transformed to a percentage overlap scale (ranging from $0-100 \%$ ), with $100 \%$ corresponding to a $\mathrm{U}$ statistic of 0.5 ). In addition, analysis of variance (ANOVA) was performed to corroborate the results.

For the end-points of FEV1 and average daily $\beta$-agonist use, change (or per cent change) from prerandomisation baseline was analysed. An ANOVA was used to compare the treatment means for all endpoints except the percentage of patients with asthma attacks, which was compared by logistical regression.

Compliance to study medication was assessed from patient-reported daily diary cards and represents the average of tablet (montelukast or placebo) and inhaler (beclomethasone or placebo) compliance.

\section{Power}

The sample size of this study, with 267 patients per active treatment group, was chosen so that the study had $95 \%$ probability to demonstrate an overlap of $\geqslant 80 \%$, with a lower limit of the $95 \%$ CI (in the distribution of patient responses for asthma control day) $>70 \%$.

\section{Results}

\section{Patients}

A total of 730 randomly allocated patients (479 females and 251 males) were randomised into the study (table 1).
Table 1. - Patient characteristics at baseline

\begin{tabular}{lccc}
\hline Characteristic & Placebo & Montelukast & Beclomethasone \\
\hline Subjects n & 103 & 313 & 314 \\
Age yrs & $35.5 \pm 14.6$ & $35.9 \pm 14.9$ & $35.5 \pm 15.0$ \\
Duration of & $18.7 \pm 13.1$ & $19.0 \pm 12.2$ & $18.2 \pm 12.4$ \\
$\quad$ asthma yrs & $2.15 \pm 0.64$ & $2.20 \pm 0.66$ & $2.25 \pm 0.65$ \\
FEV1 L & $68 \pm 12$ & $69 \pm 12$ & $68 \pm 11$ \\
FEV1 \% pred & $5.5 \pm 3.9$ & $5.2 \pm 3.7$ & $5.1 \pm 3.3$ \\
Daily $\beta$-agonist $^{\text {use puffs·day }}{ }^{-1}$ & & & \\
\hline
\end{tabular}

Data are presented as mean \pm SD; FEV1: forced expiratory volume in one second.

Of these patients, $679(93 \%)$ completed the study. Altogether, 51 patients discontinued the study prematurely $(9.7,7.0$ and $6.1 \%$ in the placebo, montelukast and beclomethasone groups, respectively), six because of a clinical adverse experience (three each in the placebo and beclomethasone groups), three because of a laboratory adverse experience (one in the montelukast and two in the beclomethasone groups) and 42 because of other reasons, such as protocol deviation, lost to follow-up, or withdrawal of consent $(7,21$ and 14 patients in the placebo, montelukast and beclomethasone groups, respectively). The per cent patient compliance (mean \pm SD) for placebo, montelukast and beclomethasone was $98.0 \pm 3.4,98.4 \pm 3.8$ and $98.1 \pm 3.5$, respectively.

\section{Efficacy results}

The overlap in per cent asthma control days between the montelukast and beclomethasone treatment groups was 89\% (95\% CI: 80.1-98.3); fig. 1).

Mean per cent of asthma control days are shown in table 2. The mean per cent of asthma control days in both the montelukast (50.7) and beclomethasone (57.9) groups was significantly higher $(\mathrm{p}<0.05)$ than that in

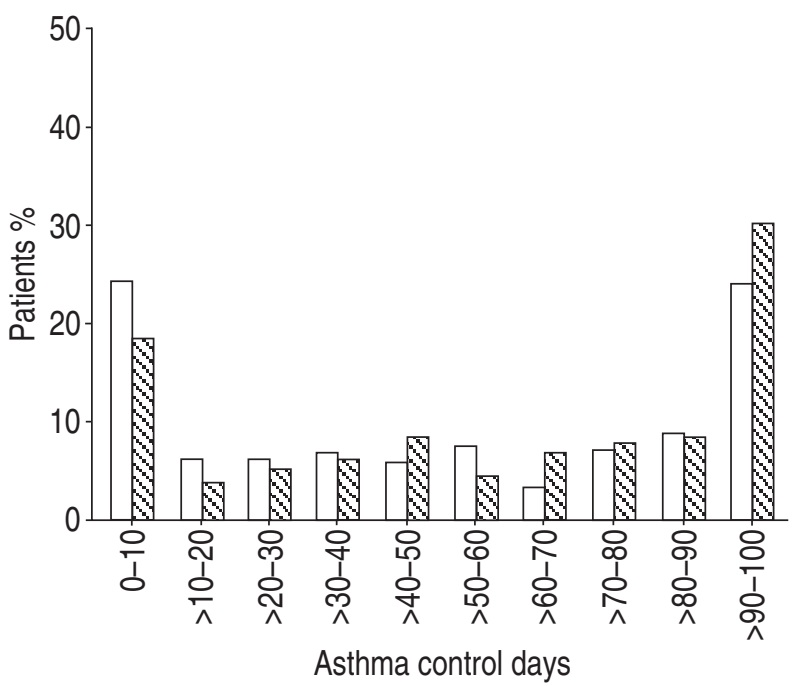

Fig. 1.-Overlap of distribution of $\%$ of asthma control days among patients receiving montelukast $(\square) 10 \mathrm{mg}$ orally once daily at bedtime or inhaled beclomethasone $(\mathbb{\mathbb { Q }}) 200 \mu \mathrm{g}$ twice daily. 
Table 2. - Results for efficacy end-points

\begin{tabular}{lccc}
\hline End-point & Placebo & Montelukast & Beclomethasone \\
\hline $\begin{array}{c}\text { Asthma control } \\
\text { days \% }\end{array}$ & $40.0 \pm 35.8$ & $50.7 \pm 37.1^{\#}$ & $57.9 \pm 36.1^{*}$, \\
$\begin{array}{c}\text { FEV1 \% change } \\
\text { from baseline }\end{array}$ & $6.4 \pm 20.1$ & $12.1 \pm 18.7^{\#}$ & $13.9 \pm 20.8^{\bullet}$ \\
$\begin{array}{c}\text { Daily } \beta \text {-agonist } \\
\text { use \% change } \\
\text { from baseline }\end{array}$ & $-15.7 \pm 50.0$ & $-35.7 \pm 42.6^{\#}$ & $-45.7 \pm 41.6^{*}$, \\
$\begin{array}{c}\text { Patient global } \\
\text { evaluation }\end{array}$ & $1.5 \pm 1.53$ & $1.1 \pm 1.19^{\#}$ & $0.9 \pm 1.00^{*}$, \\
$\begin{array}{c}\text { Physician global } \\
\text { evaluation }\end{array}$ & $1.9 \pm 1.47$ & $1.3 \pm 1.20^{\#}$ & $1.2 \pm 1.08^{\bullet}$ \\
$\begin{array}{c}\text { Asthma attack \% } \\
\text { patients }\end{array}$ & $14.9 \pm 35.1$ & $5.5 \pm 22.8^{+}$ & $3.9 \pm 19.3^{\bullet}$ \\
\hline
\end{tabular}

Data are presented as mean $\pm \mathrm{SD}$. FEV1: forced expiratory volume in one second. *: $\mathrm{p}<0.05$ for montelukast-beclomethasone comparison; ${ }^{\#}$ : $\mathrm{p}<0.05$ for placebo-montelukast comparison; : $\mathrm{p}<0.05$ for placebo-beclomethasone comparison; ${ }^{+}: \mathrm{n}$ values for placebo, montelukast and beclomethasone were 101, 308 and 308 , respectively.

the placebo group (placebo 40.0, montelukast-placebo difference 10.7, beclomethasone-placebo difference 17.9). The mean difference in per cent asthma control days favoured beclomethasone over montelukast (difference 7.2, $\mathrm{p}<0.05$ ).

Mean baseline values of FEV1 were comparable among the three treatment groups (table 1). The overlap in patient response for change from baseline in FEV1 (L) between montelukast and beclomethasone was 96\% (95\% CI: 86.6-100.0) (fig. 2).

There was no significant difference between the active treatment groups in per cent change from baseline in FEV1 (table 2). Both treatments produced significantly $(p<0.05)$ greater improvement in FEV1 when compared to placebo: the mean difference in change from baseline between placebo and montelukast was $0.11 \mathrm{~L}$ and between placebo and beclomethasone was $0.16 \mathrm{~L}$.

The per cent asthma control days (mean \pm SD) for patients with a baseline $\%$ pred FEV 1 of $\leqslant 60 \%$ were $30.3 \pm 33.6,39.9 \pm 37.2$ and $45.3 \pm 35.1$ for the placebo, montelukast and beclomethasone groups, respectively. The pairwise differences in means in per cent asthma control days between treatment groups (and the corresponding $95 \%$ CIs) were: montelukast placebo $9.6 \%$ (-6.5-25.7); beclomethasone placebo 14.9\% (-1.0-30.8) and montelukast beclomethasone $-5.3 \%$ (-17.3-6.7). The per cent asthma control days for patients with a baseline $\%$ pred FEV1 of $60-80 \%$ were $45.0 \pm 34.3$, $51.8 \pm 37.0$ and $60.1 \pm 36.3$. The pairwise differences in means in per cent asthma control days between treatment groups (and the corresponding 95\% CIs) were: montelukast placebo $6.8 \%(-4.02-17.6)$; beclomethasone placebo $15.1 \%(4.2-25.9)$ and montelukast beclomethasone $-8.3 \%(-15.6-1.0)$. The per cent asthma control days for patients with a baseline $\%$ pred FEV1 of $\geqslant 80 \%$ were $39.1 \pm 41.8,60.2 \pm 35.0$ and $67.5 \pm 32.5$. The pairwise differences in means in per cent asthma control days between treatment groups (and the corresponding 95\% CIs) were: montelukast placebo $21.0 \%$ (2.2-39.9); beclomethasone placebo

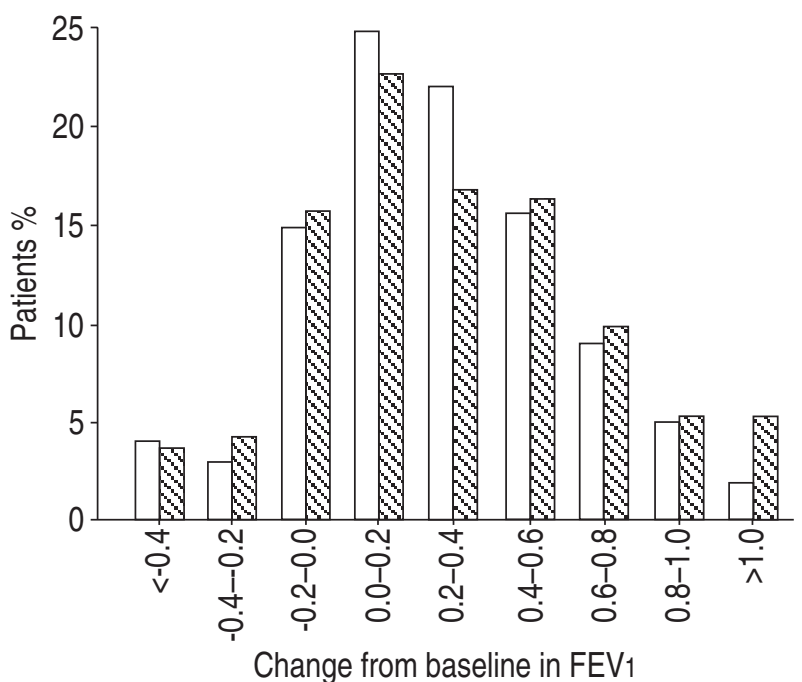

Fig. 2.-Overlap of distribution of change from baseline in forced expiratory volume in one second $\left(\mathrm{FEV}_{1}\right) \mathrm{L}$ among patients receiving montelukast $(\square) 10 \mathrm{mg}$ orally once daily at bedtime or inhaled beclomethasone $(\mathbb{\otimes}) 200 \mu \mathrm{g}$ twice daily.

$28.4 \%(9.5-47.3)$ and montelukast beclomethasone $-7.4 \%(-21.1-6.3)$.

Mean daily $\beta$-agonist use was comparable at baseline among the three treatment groups (table 1). During the study, the reduction in mean daily $\beta$-agonist use as per cent change from baseline was significantly greater for beclomethasone-treated patients than for montelukast-treated patients (mean difference $10.0 \%, \mathrm{p}<0.05$ ). Reductions in mean daily $\beta$-agonist use were significantly greater in both the montelukast and beclomethasone groups than in the placebo group (table 2). The overlap in the per cent of days between montelukast and beclomethasone in salbutamol use $(\leqslant 2$ puffs per day) was $88.4 \%$ (95\% CI: 79.3-97.5).

Improvements in both the montelukast and beclomethasone groups were significantly greater $(\mathrm{p}<0.05)$ than in the placebo group in patient and physician global assessments (table 2). Although the beclomethasone group showed a larger improvement in mean scores for the patient global evaluation than the montelukast group, there was no significant difference in mean scores for the physician global evaluation between the two groups.

There were 15 out of $101(15 \%), 17$ out of $308(6 \%)$ and 12 out of $308(4 \%)$ patients in the placebo, montelukast and beclomethasone groups, respectively, who experienced at least one asthma attack (table 2). There was no significant difference between the montelukast and beclomethasone groups in the per cent of patients suffering an asthma attack. The per cent of patients experiencing an attack in the montelukast and beclomethasone groups was significantly lower $(\mathrm{p}<0.05)$ than that in the placebo group.

\section{Safety results}

Adverse experiences were observed more frequently in patients on placebo $(54 \%)$ than those taking either 
montelukast $(39 \%)$ or beclomethasone $(42 \%)$. The most frequently reported adverse experiences were headache $(17,10$ and $11 \%$ in the placebo, montelukast and beclomethasone groups, respectively), upper respiratory infection (7, 7 and $10 \%$, respectively), and asthma exacerbations (18, 7 and 4\%, respectively). A higher percentage of patients discontinued because of adverse experiences in the placebo group $(2.9 \%)$ compared with the montelukast $(0 \%)$ and beclomethasone groups $(1 \%)$. Laboratory adverse experiences were rare and similar in frequency among treatment groups. Alanine aminotransferase elevations were the most frequent adverse experience, which occurred in none of the patients in the placebo group and one $(0.3 \%)$ and four $(1.3 \%)$ of the patients in the montelukast and beclomethasone groups, respectively. The only discontinuations due to laboratory adverse experiences were positive serum pregnancy tests (three patients).

\section{Discussion}

The intent of this study was to investigate asthma control achieved by two different therapies on a clinically relevant end-point, the asthma control day, and to compare the response distribution between groups. The definition of an asthma control day in this study combined four parameters that are commonly measured in studies of asthma therapy, namely, daily use of $\beta$-agonist, night-time awakenings, use of oral corticosteroid rescue and need for unscheduled asthma-related medical care. This definition is similar to that of the episode-free day proposed by SCULPHER and BUXTON [17] as a composite effectiveness measure for use in a cost-effectiveness analysis. An episode-free day was defined as one during which there is an absence of asthma attack, need for rescue medication, sleep disturbance caused by asthma or adverse event. This end-point represents the desired goal of consensus guidelines for treating asthma [10, 11]: a day when asthma symptoms are minimal and there is limited or no need for the use of rescue medication.

For the asthma control day end-point, the prespecified primary analysis in this study was the per cent overlap in the distribution of patient responses between the montelukast and beclomethasone treatment groups. This approach was specifically chosen to obtain new and relevant clinical information not available from comparisons of mean or median responses. The overlap analysis allows for a more complete determination of clinical comparability because the response range to therapy of the entire population is taken into account. Thus, a comparison of the relative effects requires the entire range of patient response, and therefore the use of individual patient responses, to be included in the analysis [12].

It was found that treatment for 6 weeks with either oral montelukast or inhaled beclomethasone produced a distribution of patient response to treatment that was similar in the montelukast and beclomethasone groups for both the percentage of asthma control days and FEV1. The mean per cent of asthma control days was significantly higher among patients treated with beclomethasone $(57.9 \%)$ than with those treated with montelukast (50.7\%); however, values for FEV1 (mean per cent change from baseline) were similar between the montelukast and beclomethasone groups (12.1 and $13.9 \%$, respectively). Both therapies showed significantly greater improvement than placebo in multiple parameters of asthma control.

Comparisons of the response distribution have been previously applied in other areas of clinical research, such as pharmacokinetics and vaccine efficacy, as well as in other areas of investigation. GASTwIRTH [20] noted that examination of distribution data leads to conclusions different from those derived from median values; for example, in evaluating the earnings differential between males and females, an improvement (decrease) in sex-based income discrepancy was identified when evaluating median data but showed that the discrepancy was in fact increasing on the basis of the overlap of distribution. It was concluded that the median data tended to overestimate the effect. Similarly, in the present study, there was a clinically important degree of overlap $(>85 \%)$ in the response of patients treated with either active therapy despite a difference in mean percentage of asthma control days that favoured inhaled beclomethasone.

The distribution plot also provides valuable information about the variability and the range of individual patient responses. In this study, both active therapies produced a similar range of responses for FEV1 as well as for percentage of asthma control days, a finding that suggests these drugs are similar in effect in this regard.

Both montelukast and beclomethasone were generally well tolerated and the incidence and types of adverse experiences were similar in the two groups. The overall incidence of clinical and laboratory adverse events in these two groups was lower than that in the placebo group. Asthma exacerbations occurred more frequently in the placebo group $(18,7$ and $4 \%$ of patients affected in the placebo, montelukast and beclomethasone groups, respectively).

Study medications were administered at a constant dosage throughout the treatment period and were allowed to be administered either with or without a spacer device according to the wishes of the patient and physician. This was done to simulate real world conditions and to provide a comparison of treatments as they are used in practice [22]. A dose of beclomethasone $(200 \mu \mathrm{g}$ twice daily) that is within the recommended starting-dose range $(200-500 \mu \mathrm{g})$ of the Global Initiative for Asthma guidelines was chosen [11]. In addition, Chanez et al. [23] showed that both high $(800 \mu \mathrm{g})$ and standard $(200 \mu \mathrm{g})$ initial doses of budesonide were equally effective in controlling symptoms and improving lung function in mildto-moderate asthma. The dose of montelukast is the labelled dose for adults (10 mg once daily), as established in dose-ranging studies $[24,25,26]$. In patients approximating GINA guidelines for mild patients, montelukast has demonstrated treatment benefit [27].

In conclusion, the distribution and overlap of patient responses (as assessed by asthma control days and change from baseline in forced expiratory volume in one second) indicate that during 6 weeks of therapy there is a generally similar distribution of 
response seen with montelukast and inhaled beclomethasone as administered in the trial and that the approach utilising overlap distributions to compare treatments is clinically useful and deserves wider application in the context of clinical trials.

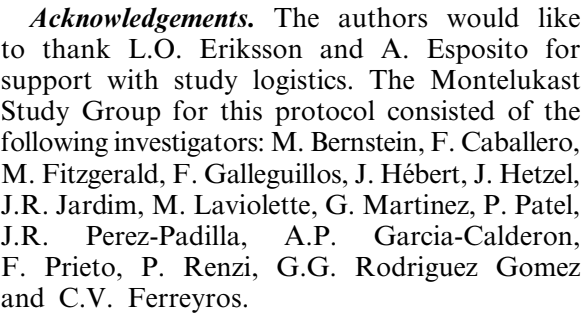

\section{References}

1. Woolcock AJ, Peat JK. Evidence for the increase in asthma worldwide. In: The rising trends in asthma. Ciba Foundation Symposium 206. Chichester, John Wiley and Sons, 1997; pp. 122-139.

2. Samuelsson B. Leukotrienes: mediators of immediate hypersensitivity reactions and inflammation. Science 1983; 220: 568-575.

3. Drazen JM, Austen KF, Lewis RA, et al. Comparative airway and vascular activity of leukotriene C-1 and $\mathrm{D}$ in vivo and in vitro. Proc Natl Acad Sci USA 1980; 77: 4345-4358.

4. Marom Z, Shelhamer JH, Bach MK, Morton DR, Kaliner M. Slow-reacting substances, leukotriene C4 and D4 increase the release of mucus from human airways. Am Rev Respir Dis 1982; 126: 449-451.

5. Barnes NC, Piper PJ, Costello JF. Comparative actions of inhaled leukotriene C4, leukotriene D4 and histamine in normal subjects. Thorax 1984; 39: 500-504.

6. Reiss TF, Chervinsky P, Dockhorn RJ, Shingo S, Seidenberg B, Edwards TB. Montelukast, a once daily leukotriene receptor antagonist, in the treatment of chronic asthma. A multicenter, randomized, doubleblind trial. Arch Intern Med 1998; 158: 1213 1220.

7. Knorr B, Matz J, Bernstein JA, et al. Montelukast for chronic asthma in 6- to 14-year-old children: A randomized, double-blind trial. JAMA 1998; 279: $1181-1186$.

8. Malmstrom K, Rodriguez-Gomez G, et al. Oral montelukast, inhaled beclomethasone, and placebo for chronic asthma. A randomized, controlled trial. Montelukast/Beclomethasone Study Group. Ann Intern Med 1999; 130: 487-495.

9. Löfdahl C-G, Reiss TF, Leff JA, et al. Randomised, placebo controlled trial of effect of a leukotriene receptor antagonist, montelukast, on tapering inhaled corticosteroids in asthmatic patients. BMJ 1999; 319: 87-90.

10. National Asthma Education and Prevention Program.
Expert panel report 2: guidelines for the diagnosis and management of asthma. NIH Publication No. 97-4051. Bethesda MD, National Institutes of Health, 1997.

11. Global Initiative for Asthma. 1998. Pocket guide for asthma management and prevention. NIH Publication No. 96-3659B. Bethesda, MD, National Institutes of Health, 1998.

12. Stine RA, Heyse JF. Non-parametric estimates of overlap. Stat Med 2001; 20: 215-236.

13. United States Department of Health and Human Services. In vivo bioequivalence studies based on population and individual bioequivalence approaches (draft: guidance). October 1997.

14. American Thoracic Society. Standardization of spirometry, 1994 update. Am J Respir Crit Care Med 1995; 152: $1107-1136$.

15. Malmstrom K, Peszek I, Botto A, Lu S, Enright PL, Reiss TF. Quality assurance of asthma clinical trials. Controlled Clin Trials 2002; 23: 143-156.

16. Santanello NC, Barber BL, Reiss TF, Friedman BS, Juniper EF, Zhang J. Measurement characteristics of two asthma symptom diary scales for use in clinical trials. Eur Respir J 1997; 10: 646-651.

17. Sculpher MJ, Buxton MJ. The episode-free day as a composite measure of effectiveness: an illustrative economic evaluation of formoterol versus salbutamol in asthma therapy. Pharmacoeconomics 1993; 4: 345352.

18. Selvin S. Statistical analysis of epidemiologic data. New York, Oxford University Press, 1991.

19. Lehmann EL. Nonparametric methods based on ranks. San Francisco, Holden-Day, 1975.

20. Gastwirth JL. Statistical measures of earnings differentials. Am Statistician 1975; 29: 32-35.

21. Conover WJ. Practical nonparametric statistics. New York, John Wiley \& Sons, 1971.

22. Schwartz D, Lellouch J. Explanatory and pragmatic attitudes in therapeutic trials. J Chronic Dis 1967; 20: 637-648.

23. Chanez P, Karlstrom R, Godard P. High or standard initial dose of budesonide to control mild-to-moderate asthma? Eur Respir J 2001; 17: 856-862.

24. Altman LC, Munk Z, Seltzer J, et al. A placebocontrolled, dose-ranging study of montelukast, a cysteinyl leukotriene-receptor antagonist. J Allergy Clin Immunol 1998; 102: 50-56.

25. Noonan MJ, Chervinsky $\mathrm{P}$, Brandon $\mathrm{M}$, et al. Montelukast, a potent leukotriene receptor antagonist, causes dose-related improvements in chronic asthma. Eur Respir J 1998; 11: 1232-1239.

26. Bronsky EA, Kemp JP, Zhang J, Guerreiro D, Reiss TF. Dose-related protection of exercise bronchoconstriction by montelukast, a cysteinyl leukotrienereceptor antagonist, at the end of a once-daily dosing interval. Clin Pharmacol Ther 1997; 62: 556-561.

27. Barnes N, Wei LX, Reiss TF, et al. Analysis of montelukast in mild persistent asthmatic patients with near-normal lung function. Respir Med 2001; 95: 379386. 